\title{
Improved accelerated breath-hold radial cine image reconstruction by acquiring additional free-breathing data between breath-holds
}

\author{
Seunghoon Nam ${ }^{1,3^{*}}$, Mehmet Akcakaya ${ }^{1}$, Yongjun Kwak ${ }^{1,3}$, Beth Goddu' ${ }^{1}$ Kraig V Kissinger ${ }^{1}$, Warren J Manning ${ }^{1,2}$, \\ Vahid Tarokh ${ }^{3}$, Reza Nezafat ${ }^{1}$
}

From 15th Annual SCMR Scientific Sessions

Orlando, FL, USA. 2-5 February 2012

\section{Background}

Evaluation of cardiac function is clinically performed using multi-slice breath-hold (BH) acquisition, in which patient breathes for a period of 40-60 seconds between a $\mathrm{BH}$ of 10-12 seconds. This results in suboptimal data acquisition efficiency. In this study, we propose to take advantage of the time between multiple $\mathrm{BHs}$ to acquire additional free-breathing (FB) data that can be used in the reconstruction of undersampled $\mathrm{BH}$ acquisition without increasing the total scan time.

\section{Methods}

Figure 1 shows the proposed data acquisition strategy. An undersampled BH acquisition is followed by a fullysampled FB acquisition during the resting period between $\mathrm{BH}$ acquisitions when the patients were instructed to breathe normally. The undersampled radial cine data were reconstructed using compressed sensing (CS) [1]. Each cardiac phase image was sparsified by subtracting the FB image with the same cardiac phase for successful CS reconstruction. Each cardiac phase image is reconstructed by an iterative CS algorithm which minimizes the objective function $\left\|\boldsymbol{A} \boldsymbol{m}_{i}-\boldsymbol{y}_{\boldsymbol{i}}\right\|_{2}{ }^{2}$ $+\lambda_{1}\left\|\boldsymbol{\Psi} \boldsymbol{m}_{i}\right\|_{1}+\lambda_{2}\left\|\boldsymbol{m}_{i}-\boldsymbol{m}_{\mathrm{FB}, i}\right\|_{1}$, where $\boldsymbol{m}_{i}$ denotes the $i$-th cardiac phase image, $\boldsymbol{y}_{i}$ denotes the undersampled radial k-space measurement for $i$-th cardiac phase, $\boldsymbol{A}$ is the radial acquisition matrix, $\boldsymbol{\Psi}$ is a sparsifying transform matrix, and $\boldsymbol{m}_{\mathrm{FB}, i}$ is the $i$-th cardiac phase image from the fully-sampled FB acquisitions. The fully-sampled FB images are reconstructed by gridding algorithm [2]. The data were acquired on 1.5T Philips scanner with ECG- gated SSFP sequence using 5-channel cardiac-coil array. The following parameters were used: $\mathrm{FOV}=(320 \mathrm{~mm})^{2}$, spatial resolution $=(1.7 \mathrm{~mm})^{2}, \mathrm{TR} / \mathrm{TE} / \alpha=3.1 / 1.5 / 55^{\circ}$, temporal resolution $=34 \mathrm{~ms}$. All $\mathrm{BH}$ and $\mathrm{FB}$ acquisitions were acquired fully-sampled, and the $\mathrm{BH}$ acquisition was retrospectively undersampled. Four FB data set were acquired for each $\mathrm{BH}$ dataset and averaged to get $\boldsymbol{m}_{\mathrm{FB}, i}$.

\section{Results}

Figure 2 shows example cardiac phase images reconstructed by the proposed CS algorithm, conventional CS algorithm which utilizes $x-f$ space sparsity [3], and the gridding algorithm. The $\mathrm{BH}$ data was undersampled to have $33 \%$ and $20 \%$ of projections from fully-sampled data. The proposed method has less streaking artifacts, sharper blood-myocardium borders and improved image qualities.

\section{Conclusions}

We demonstrated the feasibility of CS reconstruction for undersampled radial cine imaging utilizing the $\mathrm{FB}$ data acquired during the resting period between $\mathrm{BH}$ acquisitions. The results show that the CS reconstruction improves the image quality and can be used either to reduce the $\mathrm{BH}$ duration or increase the spatio-temporal resolution of cine imaging. Further studies to evaluate global cardiac function in patients are needed to validate the clinical accuracy of the proposed technique.

\section{Funding}

NIH R01EB008743-01A2.

${ }^{1}$ Medicine, Beth Israel Deaconess Medical Center, Boston, MA, USA

Full list of author information is available at the end of the article

(C) 2012 Nam et al; licensee BioMed Central Ltd. This is an open access article distributed under the terms of the Creative Commons Attribution License (http://creativecommons.org/licenses/by/2.0), which permits unrestricted use, distribution, and reproduction in any medium, provided the original work is properly cited. 


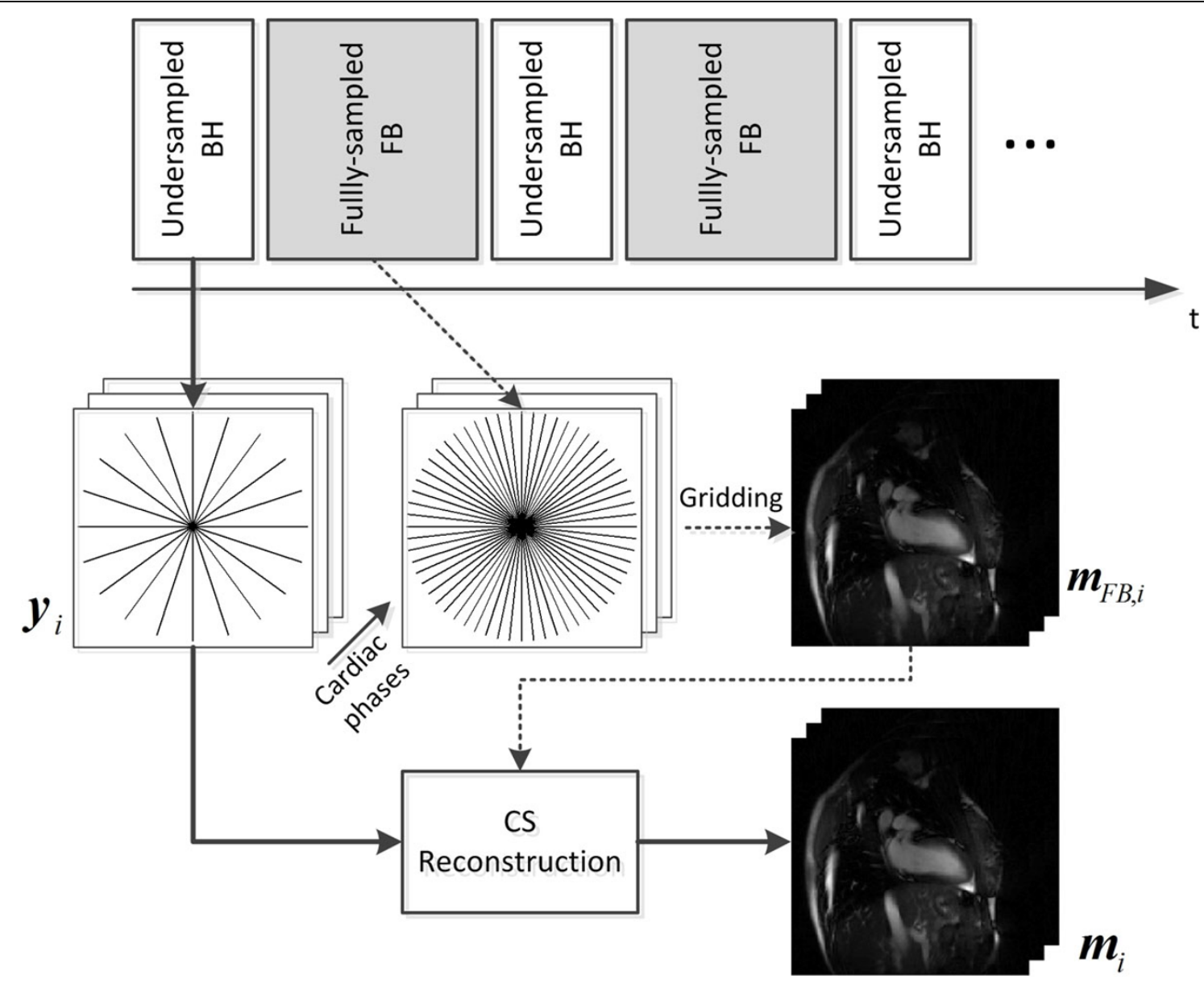

Figure 1 Acquisition of $\mathrm{BH}$ and $\mathrm{FB}$ radial cine data and the CS reconstruction.

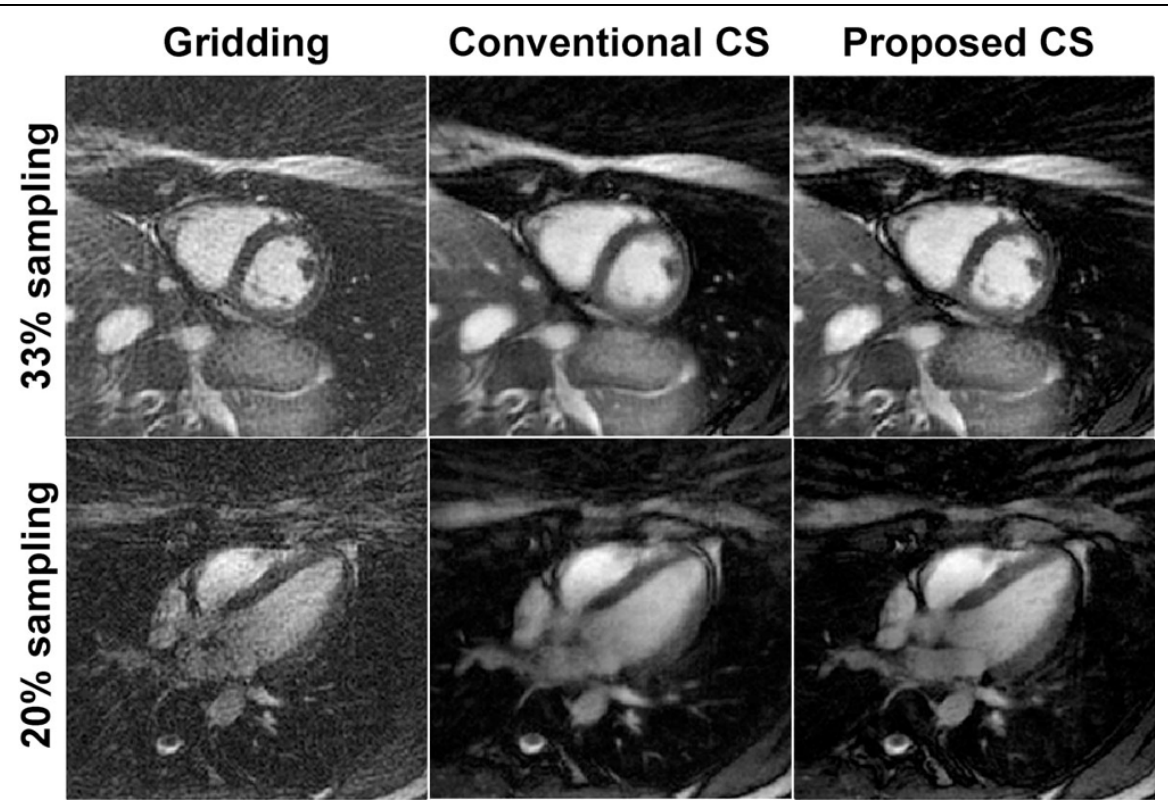

Figure 2 Example cardiac phase images of 33\% and 20\% sampling. The proposed CS reconstructions exhibit less streaking artifacts, improved sharpness and improved image qualities. 


\section{Author details}

'Medicine, Beth Israel Deaconess Medical Center, Boston, MA, USA.

${ }^{2}$ Radiology, Beth Idrael Deaconess Medical Center, Boston, MA, USA. ${ }^{3}$ School

of Engineering and Applied Sciences, Harvard University, Cambridge, MA,

USA.

Published: 1 February 2012

\section{References}

1. Lustig : MRM. 2007.

2. O'Sullivan : IEEE TMI. 1985.

3. Gamper : MRM. 2008.

doi:10.1186/1532-429X-14-S1-P269

Cite this article as: Nam et al.: Improved accelerated breath-hold radial

cine image reconstruction by acquiring additional free-breathing data

between breath-holds. Journal of Cardiovascular Magnetic Resonance 2012

14(Suppl 1):P269.

Submit your next manuscript to BioMed Central and take full advantage of:

- Convenient online submission

- Thorough peer review

- No space constraints or color figure charges

- Immediate publication on acceptance

- Inclusion in PubMed, CAS, Scopus and Google Scholar

- Research which is freely available for redistribution

Submit your manuscript at 